\title{
Acute exercise performed close to the anaerobic threshold improves cognitive performance in elderly females
}

\author{
C. Córdova1 ${ }^{1}$ V.C. Silva ${ }^{1}$, C.F. Moraes ${ }^{2}$, H.G. Simões ${ }^{1}$ and O.T. Nóbrega ${ }^{3,4}$ \\ ${ }^{1}$ Programa de Pós-Graduação stricto sensu em Educação Física e Saúde, Laboratório de Estudos em \\ Educação Física e Saúde, Universidade Católica de Brasília, Brasília, DF, Brasil \\ ${ }^{2}$ Hospital da Universidade Católica de Brasília, Brasília, DF, Brasil \\ ${ }^{3}$ Faculdade UnB em Ceilândia, Universidade de Brasília, Brasília, DF, Brasil \\ ${ }^{4}$ Programa de Pós-Graduação stricto sensu em Gerontologia, Laboratório de Imunogerontologia, \\ Universidade Católica de Brasília, Brasília, DF, Brasil \\ Correspondence to: O.T. Nóbrega, Faculdade UnB em Ceilândia, Universidade de Brasília, QNN 14 AE \\ CEI-SUL, 72220-140 Brasília, DF, Brasil \\ Fax: +55-61-3376-6042. E-mail: nobrega@pq.cnpq.br
}

\begin{abstract}
The objective of the present study was to compare the effect of acute exercise performed at different intensities in relation to the anaerobic threshold (AT) on abilities requiring control of executive functions or alertness in physically active elderly females. Forty-eight physically active elderly females (63.8 \pm 4.6 years old) were assigned to one of four groups by drawing lots: control group without exercise or trial groups with exercise performed at 60,90 , or $110 \%$ of AT (watts) and submitted to 5 cognitive tests before and after exercise. Following cognitive pretesting, an incremental cycle ergometer test was conducted to determine AT using a fixed blood lactate concentration of $3.5 \mathrm{mmol} / \mathrm{L}$ as cutoff. Acute exercise executed at $90 \%$ of AT resulted in significant $(P<0.05$, ANOVA) improvement in the performance of executive functions when compared to control in 3 of 5 tests (verbal fluency, Tower of Hanoi test (number of movements), and Trail Making test B). Exercising at $60 \%$ of AT did not improve results of any tests for executive functions, whereas exercise executed at $110 \%$ of AT only improved the performance in one of these tests (verbal fluency) compared to control. Women from all trial groups exhibited a remarkable reduction in the Simple Response Time (alertness) test $(P=0.001)$. Thus, physical exercise performed close to AT is more effective to improve cognitive processing of older women even if conducted acutely, and using a customized exercise prescription based on the anaerobic threshold should optimize the beneficial effects.
\end{abstract}

Key words: Aging; Alert; Anaerobic threshold; Exercise; Executive functions; Response time test

Research supported by UCB (SIGEP \#01/2005), UnB (DPP/UnB \#01/2008), FAPDF (\#193.000.309/2007 and \#193.000.449/ 2008), and CNPq (\#402699/2007-6).

Received June 15, 2008. Accepted February 18, 2009

\section{Introduction}

The rapid growth of the elderly population in the world, and particularly in Brazil (1), has drawn increasing attention to intervention strategies to improve the quality of life of this group. Physical exercise has been broadly recommended as a major non-pharmacological measure to prevent several dysfunctions associated with aging, including those related to cognitive performance. However, most investigations addressing exercise and cognitive performance focus on chronic exercise, whereas only a few have assessed the effects of acute physical exercise on mental performance in the elderly, with contradictory results $(2,3)$.

Studies using animal models have suggested that, in addition to increasing the number of cortical capillaries and the levels of brain-derived neurotrophic factor, chronic 
aerobic exercise may also stimulate neurogenesis and synaptogenesis and improve learning and cognitive function $(4,5)$. These observations are scientifically relevant, as they suggest a neurobiological basis for understanding cognitive plasticity in humans.

Although the results of previous studies have suggested that older adults who exercise regularly tend to benefit from an overall slower decline in cognitive function and have dementia less often (6), there is no agreement concerning what type of cognitive processing is more susceptible to the positive effects of aerobic exercise $(2,7)$. For instance, studies involving tests that require permanent monitoring of the frontal/prefrontal regions of the brain indicate that aerobic exercise improves executive functions $(2,3,8)$. In contrast, studies using tests in which continuous alertness is required report that processing speed is enhanced $(9,10)$.

In view of these results, consensual criteria should be established regarding the effects of different types of exercise programs on cognition. Several investigators $(2,7$, $11,12)$ have suggested that the influence of exercise protocols should be studied using systematic methods, with special emphasis on the dose-response relationship between physical activity and cognition $(13,14)$. In this context, blood lactate concentration has gained significance as a useful physiologic parameter in the prescription of physical exercise intensity (15). By measuring blood lactate, researchers are able to identify the exercise intensity at which the anaerobic threshold occurs, which in turn sets the moderate and high intensity ranges (16).

To our knowledge, few studies have investigated the effects of acute exercise at different intensities on abilities requiring control of executive functions or alertness in physically active elderly females, and with exercise intensity normalized according to the anaerobic threshold of each individual. The objective of the present study was to determine the effects of acute aerobic exercise performed at different intensities on abilities requiring control of executive functions or alertness in physically active elderly females.

\section{Material and Methods}

\section{Subjects}

The sample consisted of 48 women living in the urban outskirts of the Brazilian Federal District, aged 60 years or older, and enrolled in the Health Promotion Project for the Elderly at Universidade Católica de Brasília (UCB), Brasília, DF, Brazil (17-19). This particular study included older women who declared in more than one medical consultation to have been regular exercisers (muscle stretching exercises and walking at least twice a week) for a minimum of 12 months. Medical examinations were conducted to exclude individuals with active clinical signs or a history of diabetes mellitus, uncontrolled blood pressure, cerebral vascular diseases, and orthopedic/muscular-skeletal limitations. A Brazilian Portuguese version of the Beck Depression Inventory (BDI) (20) was administered to identify depression symptoms, whereas the full version of the Brazilian version of the Mini-Mental State Examination (MMSE) (21) was used for cognitive tracking, so that depression or dementia could be ruled out. In view of the educational and cultural heterogeneity of the Brazilian population and the goals of the study, cutoff scores for the MMSE and BDI were set at 26 and 15 points, respectively. None of the participants used psychoactive drugs such as antidepressants, antipsychotics and neuroleptics. The study protocol was approved by the University Ethics Committee. Each subject signed a written informed consent form.

\section{Determination of the anaerobic threshold}

The incremental cycle ergometer (Model Ergomedic 828, Monark, Sweden) approach was used to determine the anaerobic threshold (AT), defined as a blood lactate concentration of $3.5 \mathrm{mmol} / \mathrm{L}$, as well as for indirect determination of $\mathrm{VO}_{2}$ peak $(22,23)$. To determine AT, each participant underwent a cycle ergometer test consisting of two 5min exercise periods at progressive intensities with a 10min rest between the two rounds. Exercise intensities were individually set according to a 15-point rating of perceived exertion (RPE) (24). Exercise intensity had an RPE of 1012 in the first round and an RPE of 14-16 (above the AT) in the second round.

Capillary blood samples were colleted at rest both before exercise and at the end of each stage at the 1st, 3rd, and 5 th $\mathrm{min}$ of the rest interval. Blood lactate concentrations were determined electroenzymatically (YSI 1500 S sport lactate analyzer, USA) and the exercise load (watts) corresponding to the $3.5 \mathrm{mmol} / \mathrm{L}$ lactate threshold was determined by means of linear interpolation.

$\mathrm{VO}_{2}$ peak was estimated using the submaximal protocol of Astrand et al. $(22,23)$. Participants pedaled for 5 min at a rate of $50 \mathrm{rev} / \mathrm{min}$ (exercise intensity equivalent to 14$16 \mathrm{RPE}$ ). Estimated $\mathrm{VO}_{2}$ peak was computed on the basis of the equation $\mathrm{VO}_{2}$ peak $=(198-72 / \mathrm{HR}-72) \times \mathrm{VO}_{2}$ load $\left(\mathrm{VO}_{2}\right.$ load L/min $=0.014 \times$ load (watts) +0.129$)$, with adjustment for age. Heart rate (HR) was measured in beats per minute (bpm), and the mean value obtained for the rates in the 4 th and 5 th min was computed.

Exercise bouts with constant or control load

Participants (48) were randomly assigned to one of 
four groups by drawing lots: control group without exercise and trial groups with exercise performed at intensities (watts) corresponding to 60,90 , or $110 \%$ of the AT. Each exercise bout on the cycle ergometer consisted of a 5-min warm-up without load followed by 20 min of exercise with the trial load. Participants in the control group were instructed to remain seated for a time equivalent to that required for the bouts ( $25 \mathrm{~min}$ ). The cognitive performance tests were administered $8 \mathrm{~min}$ after the end of the exercise to allow HR to return to a resting status (maximally $10 \%$ above each individual baseline rate). Exercise bouts were performed at least $48 \mathrm{~h}$ after AT determination.

\section{Cognitive tests}

After the collection of clinical data but before the exercise sessions, participants underwent four random pretests so they could become familiar with the test protocols. The pretests produced asymptotic learning curves suggesting that they were effective in attenuating the influence of having to learn the task for subsequent investigation of the effects of exercise on cognitive performance. All cognitive tests in this investigation have been validated in previous studies (25). Testing was conducted between 2:00 and $6: 00 \mathrm{pm}$. The following is a brief description of the tests used.

The Tower of Hanoi test for executive functions requires the ability to plan and organize strategies to attain a certain objective (26). A wood apparatus (14.76" x 6.5") was used consisting of four blocks varying in color and size. The stopwatch went off as the first movement started and was stopped when the last block was placed on the tower (s).

The Simple Response Time test is used to investigate alertness (27). Subjects were told to look at the center of a color monitor screen and, as fast as they could, press the space bar of a standard keyboard whenever a square (0.98" x 0.98") appeared at the center of the screen. The 300-millisecond long presentations were automatically randomized over the twenty trials/test. Test duration was 3 min, and the results were computed as trial means in milliseconds. Only tests with $\geq 95 \%$ success were analyzed.

The Trail Making test is a two-part test for executive functions that requires efficient cognitive flexibility (28). In part $A$, each subject was instructed to use a pencil to sequentially connect numbered, randomly scattered circles on a sheet of paper. In part B, letters were added to the test, so that both series, i.e., numbers and letters (1-A, 2-B, 3-C, and so on) had to be used to form the correct sequence. Performance was assessed based on the time (seconds) spent to complete the test.
In addition to testing executive functions, the Verbal Fluency test is also used as a sensitive indicator of frontal dysfunction since it evaluates responses that primarily derive from the frontal areas of the human brain (29). Each subject was told to orally name as many animals as she could. The final score was computed as the number of animals recalled, excluding repeated ones. When two words referred to the same animal (for instance: dog/ bitch), only the first reference was considered. The stopwatch went off upon an oral command by the tester to start the test and was stopped after $1 \mathrm{~min}$.

\section{Statistical analysis}

Normality of distribution for variables expressing the sociodemographic characteristics was previously tested using the Kolmogorov-Smirnov test. Since the distribution of educational level was non-normal, the Kruskal-Wallis test was used for comparison between groups in terms of this variable. The performance in each cognitive test was computed as the difference between means of the aftertest scoring and the scores obtained in the fourth pre-test (pre-4) for each subject. This scoring procedure is an alternative that replaces the ANCOVA pre-test (30) and represents an additional strategy for controlling the bias of having to learn the task (31). Effect sizes (Cohen's $d$ ) were calculated for each variable by dividing the mean difference by the pooled standard deviation (32).

Analyses of variance (one-way ANOVA) were used for comparison between groups. When significant differences were found, Bonferroni's multiple comparison test adjusted to a significance level of $\mathrm{P}<0.05$ was used for identification of relevant contrasts. The statistical package SPSS version 8.0 was used for data analysis.

\section{Results}

Among 70 volunteers, 48 elderly females $(63.8 \pm 4.6$ years old) met the inclusion criteria and were included in the study. The control and experimental groups had similar sociodemographic characteristics $(P>0.05$; Table 1$)$, indicating that the control group was acceptable for the purposes of the study. Besides normalization of the anaerobic threshold using lactate measurements, HR was assessed immediately after the exercise bout to confirm that the acute intervention had produced a significant physiological response. Significant differences $(P<0.001)$ were detected when the groups exercising at $60 \%$ (118.8 \pm $3.5 \mathrm{bpm}), 90 \%$ (124.6 $\pm 21.0 \mathrm{bpm})$, and $110 \%$ (139.8 \pm $26.0 \mathrm{bpm})$ of AT were compared to the control group (87.8 $\pm 13.0 \mathrm{bpm})$.

Table 2 presents the results of the cognitive tests 
applied before and after exercise to the control and trial groups. Acute exercise conducted at $90 \%$ of AT significantly improved the performance of executive functions compared to the control group in 3 (verbal fluency, Tower of Hanoi test - number of movements, and Trail Making test - part B) of the 5 tests performed. A remarkable reduction in the Simple Response Time test $(P=0.001)$ was observed in all experimental groups compared to control. Exercise performed at $110 \%$ of AT tended either to be less advantageous or to have an adverse effect.

\section{Discussion}

The results of this investigation suggest that acute physical exercise may improve alertness and the processing of executive functions in physically active elderly females. The present study represents an important new contribution to the literature since its experimental design allowed us to demonstrate that both executive functions and alertness are directly and simultaneously enhanced by an acute exercise intervention. Previous studies of the

Table 1. Sociodemographic data for the control and trial groups.

\begin{tabular}{lrrrr}
\hline & \multicolumn{3}{c}{ Exercise intensity in relation to anaerobic threshold } \\
\cline { 2 - 5 } & Control $(\mathrm{N}=12)$ & $60 \%(\mathrm{~N}=12)$ & $90 \%(\mathrm{~N}=12)$ & $110 \%(\mathrm{~N}=12)$ \\
\hline Age (years) & $62.2 \pm 4.4$ & $65.9 \pm 3.6$ & $63.8 \pm 4.9$ & $63.1 \pm 5.1$ \\
Educational level (school years) & $8.3 \pm 2.3$ & $8.8 \pm 1.6$ & $8.8 \pm 1.3$ & $8.7 \pm 2.4$ \\
MMSE (scores) & $26.9 \pm 1.4$ & $27.6 \pm 1.0$ & $27.1 \pm 1.1$ & $27.2 \pm 1.5$ \\
BDI (scores) & $8.3 \pm 2.1$ & $9.1 \pm 2.2$ & $8.4 \pm 2.8$ & $8.1 \pm 3.4$ \\
VO $_{2}$ peak (L/min) & $22.8 \pm 3.9$ & $20.6 \pm 6.2$ & $22.2 \pm 5.9$ & $23.2 \pm 4.5$ \\
BMI (kg/m $\left.{ }^{2}\right)$ & $24.0 \pm 2.4$ & $26.3 \pm 2.5$ & $24.2 \pm 3.2$ & $24.3 \pm 3.6$
\end{tabular}

Data are reported as means \pm SD. MMSE = Mini-Mental State Examination; BDI = Beck Depression Inventory; $\mathrm{VO}_{2}$ $=$ estimated $\mathrm{VO}_{2}$ peak; $\mathrm{BMI}=$ body mass index. The Kruskal-Wallis test was used to analyze educational level. Oneway ANOVA was used for all other data. There were no statistically significant differences between groups.

Table 2. Cognitive performance of elderly women exercising above and below their anaerobic threshold.

\begin{tabular}{|c|c|c|c|c|c|c|c|c|c|c|c|c|c|}
\hline & \multicolumn{3}{|c|}{ Control } & \multicolumn{3}{|c|}{$60 \%$} & \multicolumn{3}{|c|}{$90 \%$} & \multicolumn{3}{|c|}{$110 \%$} & \multirow[t]{2}{*}{$P$} \\
\hline & Pre-4 & After & ES & Pre-4 & After & ES & Pre-4 & After & ES & Pre-4 & After & ES & \\
\hline $\mathrm{SRT}(\mathrm{ms})$ & $\begin{array}{c}373.8 \pm \\
19.0\end{array}$ & $\begin{array}{c}383.2 \pm \\
18.0\end{array}$ & 0.51 & $\begin{array}{c}366.2 \pm \\
26.0\end{array}$ & $\begin{array}{c}363.8 \pm \\
26.0^{\mathrm{a}}\end{array}$ & -0.09 & $\begin{array}{c}368.8 \pm \\
20.4\end{array}$ & $\begin{array}{c}348.5 \pm \\
25.2^{\mathrm{a}, \mathrm{b}}\end{array}$ & -0.89 & $\begin{array}{c}370.2 \pm \\
22.4\end{array}$ & $\begin{array}{c}358.6 \pm \\
24.9^{a}\end{array}$ & -0.49 & 0.001 \\
\hline $\begin{array}{r}\text { Verbal fluency } \\
\text { (No. of words) }\end{array}$ & $\begin{array}{c}17.8 \pm \\
3.1\end{array}$ & $\begin{array}{l}17.1 \pm \\
3.8\end{array}$ & -0.19 & $\begin{array}{c}16.0 \pm \\
3.8\end{array}$ & $\begin{array}{c}17.6 \pm \\
4.1\end{array}$ & 0.40 & $\begin{array}{l}16.6 \pm \\
3.4\end{array}$ & $\begin{array}{c}19.8 \pm \\
2.7 \mathrm{c}\end{array}$ & 1.07 & $\begin{array}{c}17.8 \pm \\
4.8\end{array}$ & $\begin{array}{c}22.2 \pm \\
8.8^{a}\end{array}$ & 0.84 & 0.001 \\
\hline $\begin{array}{l}\text { Tower of Hanoi } \\
\text { (No. of movements) }\end{array}$ & $\begin{array}{c}19.9 \pm \\
3.9\end{array}$ & $\begin{array}{c}20.5 \pm \\
4.2\end{array}$ & 0.14 & $\begin{array}{c}18.3 \pm \\
2.4\end{array}$ & $\begin{array}{c}18.2 \pm \\
2.1\end{array}$ & -0.04 & $\begin{array}{l}19.7 \pm \\
2.5\end{array}$ & $\begin{array}{c}18.3 \pm \\
2.0^{\mathrm{a}}\end{array}$ & -0.60 & $\begin{array}{c}19.2 \pm \\
2.6\end{array}$ & $\begin{array}{c}20.0 \pm \\
6.6\end{array}$ & 0.18 & 0.001 \\
\hline Tower of Hanoi (s) & $\begin{array}{l}79.2 \pm \\
26.1\end{array}$ & $\begin{array}{l}87.4 \pm \\
32.3\end{array}$ & 0.28 & $\begin{array}{l}80.3 \pm \\
45.2\end{array}$ & $\begin{array}{l}76.4 \pm \\
43.4\end{array}$ & -0.08 & $\begin{array}{l}69.2 \pm \\
24.2\end{array}$ & $\begin{array}{l}61.0 \pm \\
18.3^{\mathrm{d}}\end{array}$ & -0.38 & $\begin{array}{c}103.5 \pm \\
66.0\end{array}$ & $\begin{array}{c}115.9 \pm \\
70.2\end{array}$ & 0.18 & 0.02 \\
\hline TMT (Part A; s) & $\begin{array}{l}78.1 \pm \\
16.1\end{array}$ & $\begin{array}{l}78.6 \pm \\
16.5\end{array}$ & 0.03 & $\begin{array}{l}69.1 \pm \\
15.9\end{array}$ & $\begin{array}{l}65.6 \pm \\
15.2\end{array}$ & -0.22 & $\begin{array}{l}76.2 \pm \\
22.7\end{array}$ & $\begin{array}{l}66.4 \pm \\
21.4\end{array}$ & -0.44 & $\begin{array}{l}69.8 \pm \\
21.2\end{array}$ & $\begin{array}{l}71.0 \pm \\
19.7\end{array}$ & 0.06 & 0.06 \\
\hline TMT (Part B; s) & $\begin{array}{c}156.8 \pm \\
43.9\end{array}$ & $\begin{array}{c}165.0 \pm \\
32.7\end{array}$ & 0.21 & $\begin{array}{c}143.2 \pm \\
39.1\end{array}$ & $\begin{array}{c}137.8 \pm \\
33.3\end{array}$ & -0.15 & $\begin{array}{c}143.4 \pm \\
47.6\end{array}$ & $\begin{array}{l}131.7 \pm \\
36.6^{\mathrm{a}, \mathrm{d}, \mathrm{e}}\end{array}$ & -0.28 & $\begin{array}{c}160.4 \pm \\
72.8\end{array}$ & $\begin{array}{c}168.6 \pm \\
71.0\end{array}$ & 0.11 & 0.001 \\
\hline
\end{tabular}

Data are reported as means \pm SD. ES = effect size; SRT = Simple Response Time test; TMT = Trail Making test. ap $<0.01$ compared to control; bP $<0.01$ compared to the group exercising at $60 \%$ of anaerobic threshold (AT); ${ }^{\mathrm{C} P}<0.05$ compared to control; ${ }^{\mathrm{d} P}<0.05$ compared to the group exercising at $110 \%$ of AT; eP $<0.05$ compared to group exercising at $60 \%$ of AT. One-way ANOVA was used for comparison between groups with Bonferroni's multiple comparison test when significant differences were found. 
effects of chronic exercise on comparable variables cannot claim to have demonstrated this relationship because the processing of executive functions and attentional responses may be the result of interdependent, incremental effects of these functions on one another during a longterm exercise program. This fact makes it difficult to establish a cause and effect relationship.

Specifically, exercise at $90 \%$ of AT $\left(\sim 50 \% \mathrm{VO}_{2}\right.$ peak) was more appropriate for optimizing the performance of executive functions (up to $16.4 \%$ ). Previous studies addressing different populations and employing different trial protocols have also demonstrated improved cognitive performance in participants submitted to intensities between $40-60 \% \mathrm{VO}_{2}$ peak $(10,11,32)$. With regard to executive function tests, our results are consistent with those of previous studies addressing the acute effects of either aerobic exercise (8) or regular programs aiming to achieve cardiovascular fitness (33).

According to Chmura et al. (33), cortical neuroelectric activity increases with increasing intensity of physical exercise, but reaches levels that favor cognitive performance at moderate intensities. This may explain the poorer performance in the present study of the group exercising at $110 \%$ of AT ( $60 \% \mathrm{VO}_{2}$ peak) compared to the $90 \%$ group. Furthermore, it has been suggested that exercising above AT is associated with decreased $\mathrm{pH}$ and depletion of alkaline stores (16), which may explain why in the present study exercising below the AT $(90 \%)$ tended to be more effective than exercising above the AT $(110 \%)$ in potentiating the performance of more complex cognitive tasks such as the Trail Making test - part B (Table 2). It is widely known that cerebral acidosis depresses, while alkalosis enhances, neuronal excitability mediated via voltage-gated $\mathrm{Na}^{+}, \mathrm{K}^{+}$, or $\mathrm{Ca}^{2+}$ channels, even when $\mathrm{pH}$ changes within a physiological range ( $\mathrm{pH} 6-8)$ (34)

Exercise intensity at the onset of blood lactate accumulation has long been considered to correspond to the AT. According to Heck et al. (35), the blood lactate concentration to be considered for determination of the onset of blood lactate accumulation is 3.5 and $4.0 \mathrm{mM}$ if the duration of incremental stages is about 3 and $5 \mathrm{~min}$, respectively. We chose the concentration $3.5 \mathrm{mM}$ because the aging process is associated with lower lactacidemia, especially in women (36), and a fixed concentration of $4 \mathrm{mmol} / \mathrm{L}$ may overestimate the exercise intensity required to reach AT in our subjects, as observed in a pilot study.

Particularly interesting were the results involving the alertness level of subjects when indirectly assessed via latency of responses in the Simple Response Time test. For instance, when the groups exercising close to AT are compared to the control group and to the group at $60 \%$ of
AT ( 22\% VO $\mathrm{VO}_{2}$ peak), the former groups experienced a considerable reduction in the latency of responses (up to $5.5 \%$ ). This behavior suggests that a certain level of physical stress is required to make up for the negative effects resulting from the repetitive nature of this test of alertness.

Differently from us, Hillman et al. (8) failed to detect the effect of acute physical exercise on the response time. According to these investigators, the benefits of aerobic exercise are selective for executive control functions. These discrepancies may result in part from methodological differences: Hillman et al. (8) administered the cognitive test about $48 \mathrm{~min}$ after exercise, whereas in our investigation the tests were performed about $8 \mathrm{~min}$ after the intervention. This interval was defined on the basis of pre-testing assessments showing that 8 min were sufficient to reduce the HR of each subject to baseline levels. Although there is no consensus on how physical activity close to AT improves the speed of cognitive processing, it has been suggested that the concentration of central and peripheral catecholamines in response to aerobic exercise is associated with the activation of the central nervous system (37) and with improved performance in the time of response tests (38). Considered that, interpretation of the present results can rely on the assumption that increased circulating adrenaline induced by physical activity triggers significant cognitive changes in humans. However, since the noradrenergic concentration quickly returns to baseline values, it is of key importance to identify the best time to administer tests requiring processing speed (10). It should be noted that a placebo effect is unlikely to have occurred in the present study, as the group exercising at $60 \%$ of AT did not demonstrate improved results in the battery of cognitive tests for executive functions.

Thus, we conclude that exercise conducted below AT is more effective to improve different aspects of cognitive processing in elderly women. Moreover, our results suggest that physical exercise is beneficial for cognitive performance even if conducted acutely, and that optimization based on the anaerobic threshold is suitable for a customized prescription. This study had the limitation of evaluating blood lactate solely for prescription of exercise intensity, based on the decision to minimize physical and/or psychological stress in the subjects. Since the study was carried out with a group of physically active females, the present conclusions are not necessarily valid for male or sedentary subjects or for other modalities of physical exercise. Moreover, additional studies should be conducted to compare the effects of chronic exercise at different intensities in relation to AT on the cognitive performance of elderly people.

Taken together, our results suggest that the practice of 
aerobic exercise at an elevated but not exhaustive level of intensity may be employed as a non-pharmacological measure to enhance attentional phenotypes as well as functional aspects associated with the brain's frontal/prefrontal regions in physically active older women.

\section{References}

1. Nobrega OT, Faleiros VP, Telles JL. Gerontology in the developing Brazil: achievements and challenges in public policies. Geriatr Gerontol Int 2009 (ahead of print).

2. Jedrziewski MK, Lee VM, Trojanowski JQ. Physical activity and cognitive health. Alzheimers Dement 2007; 3: 98-108.

3. Fratiglioni L, Paillard-Borg S, Winblad B. An active and socially integrated lifestyle in late life might protect against dementia. Lancet Neurol 2004; 3: 343-353.

4. Cotman CW, Berchtold NC. Exercise: a behavioral intervention to enhance brain health and plasticity. Trends Neurosci 2002; 25: 295-301.

5. Swain RA, Harris AB, Wiener EC, Dutka MV, Morris HD, Theien $\mathrm{BE}$, et al. Prolonged exercise induces angiogenesis and increases cerebral blood volume in primary motor cortex of the rat. Neuroscience 2003; 117: 1037-1046.

6. Rovio S, Kareholt I, Helkala EL, Viitanen M, Winblad B, Tuomilehto J, et al. Leisure-time physical activity at midlife and the risk of dementia and Alzheimer's disease. Lancet Neurol 2005; 4: 705-711.

7. Tomporowski PD. Effects of acute bouts of exercise on cognition. Acta Psychol 2003; 112: 297-324.

8. Hillman $\mathrm{CH}$, Snook EM, Jerome GJ. Acute cardiovascular exercise and executive control function. Int J Psychophysiol 2003; 48: 307-314.

9. Antunes HKM, Santos RF, Cassilhas R, Santos RVT, Bueno OFA, Mello MT. Reviewing on physical exercise and the cognitive function. Rev Bras Med Esporte 2006; 12: 108114.

10. Davranche K, Audiffren M. Facilitating effects of exercise on information processing. J Sports Sci 2004; 22: 419-428.

11. Colcombe SJ, Kramer AF, Erickson KI, Scalf P, McAuley E, Cohen NJ, et al. Cardiovascular fitness, cortical plasticity, and aging. Proc Natl Acad Sci U S A 2004; 101: 3316-3321.

12. Fabre $\mathrm{C}$, Chamari K, Mucci $\mathrm{P}$, Masse-Biron J, Prefaut $\mathrm{C}$. Improvement of cognitive function by mental and/or individualized aerobic training in healthy elderly subjects. Int $J$ Sports Med 2002; 23: 415-421.

13. Etnier JL, Nowell PM, Landers DM, Sibley BA. A metaregression to examine the relationship between aerobic fitness and cognitive performance. Brain Res Rev 2006; 52 : 119-130.

14. Middleton LE, Mitnitski A, Fallah N, Kirkland SA, Rockwood $\mathrm{K}$. Changes in cognition and mortality in relation to exercise in late life: a population based study. PLOS ONE 2008; 3: e3124.

15. Weltman A. The blood lactate response to exercise. Champaign: Human Kinetics; 1995

16. Simoes HG, Campbell CS, Kushnick MR, Nakamura A, Katsanos CS, Baldissera V, et al. Blood glucose threshold and the metabolic responses to incremental exercise tests with and without prior lactic acidosis induction. Eur J Appl Physiol 2003; 89: 603-611.
17. Tonet AC, Karnikowski M, Moraes CF, Gomes L, Karnikowski MG, Cordova C, et al. Association between the -174 G/C promoter polymorphism of the interleukin- 6 gene and cardiovascular disease risk factors in Brazilian older women. Braz J Med Biol Res 2008; 41: 47-53.

18. Moraes CF, Souza ER, Souza VC, Medeiros EF, Goncalves $\mathrm{TF}$, Toledo JO, et al. A common polymorphism in the renin angiotensin system is associated with differential outcome of antihypertensive pharmacotherapy prescribed to Brazilian older women. Clin Chim Acta 2008; 396: 70-75.

19. Karnikowski M, Cordova C, Oliveira RJ, Karnikowski MG, Nobrega OT. Non-alcoholic fatty liver disease and metabolic syndrome in Brazilian middle-aged and older adults. São Paulo Med J 2007; 125: 333-337.

20. Gorenstein C, Andrade L. Beck Depression Inventory: Psychometric properties of the Portuguese version. Rev Psiquiatr Clin 1998; 25: 245-250.

21. Brucki SM, Nitrini R, Caramelli $\mathrm{P}$, Bertolucci $\mathrm{PH}$, Okamoto $\mathrm{IH}$. Suggestions for utilization of the mini-mental state examination in Brazil. Arq Neuropsiquiatr 2003; 61: 777-781.

22. Åstrand P-O. Textbook of work physiology: physiological bases of exercise. 4th edn. Champaign: Human Kinetics; 2003.

23. Astrand PO, Ryhming I. A nomogram for calculation of aerobic capacity (physical fitness) from pulse rate during sub-maximal work. J Appl Physiol 1954; 7: 218-221.

24. Burkhalter N. Evaluation of Borg's perceived exertion scale in cardiac rehabilitation. Rev Lat Am Enfermagem 1996; 4: 65-73.

25. Strauss E, Sherman EMS, Spreen O. A compendium of neuropsychological tests. 3rd edn. New York: Oxford University Press; 2006.

26. Camozzato A, Chaves ML. Schizophrenia in males of cognitive performance: discriminative and diagnostic values. Rev Saúde Pública 2002; 36: 743-748.

27. Córdova C, Bravin AA, Oliveira RJ, Barros JF. Reaction time in socio-cultural adult retardates and its relationship with differences in motor development. Neurobiologia 2003; 66: 53-59.

28. Moll J, de Oliveira-Souza R, Moll FT, Bramati IE, Andreiuolo PA. The cerebral correlates of set-shifting: an fMRI study of the Trail Making test. Arq Neuropsiquiatr 2002; 60: 900-905.

29. Radanovic M, Azambuja M, Mansur LL, Porto CS, Scaff M. Thalamus and language: interface with attention, memory and executive functions. Arq Neuropsiquiatr 2003; 61: 3442.

30. Tabachnick BG, Fidel LS. Using multivariate statistics. New York: Harper Collins Publishers; 1996.

31. Wesnes K, Pincock C. Practice effects on cognitive tasks: a major problem? Lancet Neurol 2002; 1: 473.

32. Cohen J. A power primer. Psychol Bull 1992; 112: 155-159.

33. Chmura J, Nazar K, Kaciuba-Uscilko H. Choice reaction 
time during graded exercise in relation to blood lactate and plasma catecholamine thresholds. Int J Sports Med 1994; 15: 172-176.

34. Tombaugh GC, Somjen GG. Effects of extracellular pH on voltage-gated $\mathrm{Na}^{+}, \mathrm{K}^{+}$and $\mathrm{Ca}^{2+}$ currents in isolated rat $\mathrm{CA} 1$ neurons. J Physiol 1996; 493 (Part 3): 719-732.

35. Heck H, Mader A, Hess G, Mucke S, Muller R, Hollmann W. Justification of the 4-mmol/L lactate threshold. Int J Sports Med 1985; 6: 117-130.

36. Korhonen MT, Suominen H, Mero A. Age and sex differ- ences in blood lactate response to sprint running in elite master athletes. Can J Appl Physiol 2005; 30: 647-665.

37. Pagliari R, Peyrin L. Norepinephrine release in the rat frontal cortex under treadmill exercise: a study with microdialysis. $J$ Appl Physiol 1995; 78: 2121-2130.

38. Peyrin L, Pequignot JM, Lacour JR, Fourcade J. Relationships between catecholamine or 3-methoxy 4-hydroxy phenylglycol changes and the mental performance under submaximal exercise in man. Psychopharmacology 1987; 93: $188-192$ 